\title{
The Relationship of Facet Joint Orientation and Tropism with Lumbar Disc Herniation and Degenerative Spondylolisthesis in the Lower Lumbar Spine
}

\author{
Devanand Degulmadi, Bharat Dave, Ajay Krishnan, Denish Patel \\ Department of Spine Surgery, Stavya Spine Hospital and Research Institute Private Limited, Ahmedabad, India
}

\begin{abstract}
Study design: Retrospective study.
Purpose: This study aimed to clarify the relationship of both facet tropism (FT) and the sagittally aligned facet (SAF) joint with lumbar disc herniation (LDH) and degenerative spondylolisthesis (DS).

Overview of Literature: Despite several studies conducted, there is no consensus on the association of the SAF joint and FT with DH and DS.

Methods: Between June 2015 and December 2017, magnetic resonance imaging scans of 250 consecutive patients who underwent surgery for LDH and DS were analyzed. The facet angles at all the lower lumbar levels were calculated, and SAF and FT were noted. The relationship between the side of disc herniation and that of the SAF joint were also determined. Statistical analysis was performed, and the relation of SAF and FT to LDH and DS was noted.

Results: We observed a positive relationship between SAF and LDH at L4-5 and L5-S1 with a $p$-value of $0.02(<0.05)$. FT demonstrated a positive association with $\mathrm{LDH}$ at $\mathrm{L} 4-5(p=0.047)$ but not at $\mathrm{L} 3-4$ or $\mathrm{L} 5-\mathrm{S} 1$. SAF demonstrated a positive relationship with DS at L3-4 $(p<0.001)$ but not at L3-4 or L5-S1. FT demonstrated a significant relation with DS at $L 4-5(p<0.001)$, whereas no positive association was observed at L3-4 and L5-S1.

Conclusions: The L4-5 level demonstrated a significant association with SAF and FT in LDH and DS. Moreover, SAF at L5-S1 demonstrated a positive association with LDH. These findings provide useful information for future longitudinal studies to elucidate the possible causes for such phenomena.
\end{abstract}

Keywords: Sagittally aligned facet joint; Facet tropism; Lumbar disc herniation; Degenerative spondylolisthesis

\section{Introduction}

The potential significance of an asymmetrical posterior lumbar facet in the pathogenesis of low back pain was proposed by Putti [1] in 1927, who introduced the term 'facet tropism' (FT). FT was described in 1929 by Brailsford [2] as an asymmetry between the left and right vertebral (apophyseal) facet joint angles with one joint hav-

Received May 23, 2018; Revised Jun 13, 2018; Accepted Jun 17, 2018

Corresponding author: Devanand Degulmadi

Stavya Spine Hospital and Research Institute Private Limited, Mithakhali, Ellisbridge, Ahmedabad, Gujarat 380006, India

Tel: +91-79-26565757, +91-79-26565657, Fax: +91-7874444091, E-mail: drdanand@yahoo.co.in 
ing a sagittal orientation of $5^{\circ}$ or $7^{\circ}$ more than the other. Despite several studies conducted, there is no consensus on the relation between sagittally aligned facet (SAF) joint and lumbar disc herniation (LDH). The hypothesis that FT is correlated with the presence of disc herniation is still debated. Some studies have suggested that the SAF joint contributes to disc degeneration and subsequent degenerative spondylolisthesis (DS). However, the role of FT in DS is still unclear. The aim of this study was to clarify the relationship of both FT and the SAF joint with $\mathrm{LDH}$ and DS. Further, we calculated the relationship between the side of disc herniation and that of the SAF joint.

\section{Materials and Methods}

Between June 2015 and December 2017, magnetic resonance imaging (MRI) scans of 250 consecutive patients who underwent surgery for LDH and DS were analyzed. Data were classified according to the predetermined criteria, and the patients were divided into the LDH and DS groups. Only MRI scans with complete records were selected until the intended number of 250 was achieved for each group. Institutional ethical committee clearance was not required as the study was only conducted on the images.

\section{Measurement of the facet angle}

The orientation of the facet angles was measured on the axial T2-weighted MRI images using the method de- scribed by Noren et al. [3] from the third lumbar to first sacral vertebrae. MB Angle software (Markus Bader-MB software solutions ver. 5.3; MB Software Solutions, Iffezheim, Germany) was used to calculate the left and right facet joint angles. On the T2 axial image that bisected the intervertebral disc, one line was drawn in the midsagittal plane of the vertebra passing through the center of the disc and center of the base of the spinous process, and another line was drawn between the anteromedial and posterolateral edges of the superior articular facets bilaterally (Fig. 1). All facet angles were measured as the angle between the oblique line and the sagittal plane on both sides. The cutoff angles of $36^{\circ}$ at L3-4, $42^{\circ}$ at L4-5, and $45^{\circ}$ at L5-S1 were set as indicators for SAF joints. The difference between the two angles (FT) was calculated. According to the method described by Vanharanta et al. [4], moderate tropism was defined as a difference of $7^{\circ}$ to $15^{\circ}$ and severe tropism as more than $15^{\circ}$.

\section{Data and statistical analysis}

All the observations were recorded on a standard Excel sheet (Microsoft Corp., Redmond, WA, USA). The average facet angles including standard deviation were calculated at each level on both sides. The incidence of sagittal facet angles and FT based on the criteria set was noted at each level. In the $\mathrm{LDH}$ group, the distribution of the level of disc herniation and side of herniation at different levels was recorded. In addition, the association between $\mathrm{SAF}$ and FT in relation to the side of herniation at each
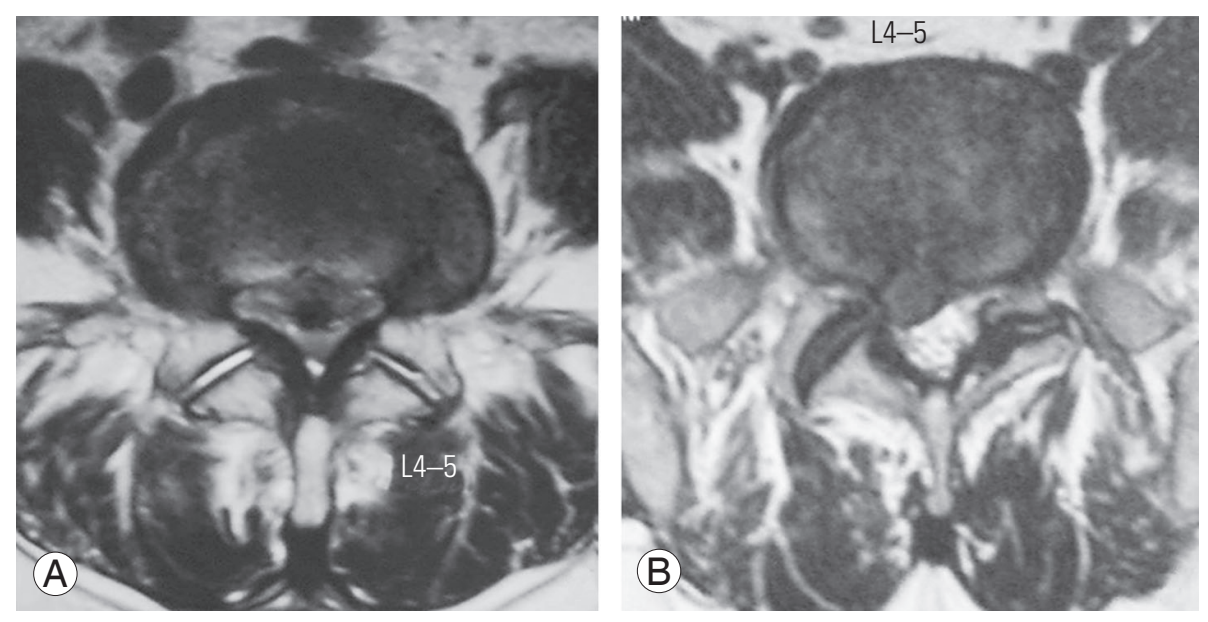

Fig. 1. (A) MRI image showing the central type of disc prolapse without facet tropism. (B) MRI image showing a classic unilateral disc prolapse with facet tropism. Note that the disc prolapse is toward the sagittal facet. MRI, magnetic resonance imaging. 
lower lumbar level was analyzed. In the DS group, a similar analysis was performed for each lower lumbar level in terms of SAF and FT. Chi-square test was used to determine the relationship between the previously mentioned criteria. A $p$-value $<0.05$ was considered significant.

\section{Results}

Of the 500 patients studied, $51 \%$ were males and $49 \%$ were females. The mean age was 49.1 years (range, 15-88 years). A total of 1,500 lumbar levels (3,000 facet angles) were considered, including L3-4, L4-5, and L5-S1. The patients were divided into two groups, the LDH group and the DS group, based on the diagnosis.

In the LDH group, 17 (6.8\%) had disc herniation at
L3-4, $135(55.2 \%)$ at L4-5, and $98(39.2 \%)$ at L5-S1. SAF at L3-4 $\left(<36^{\circ}\right)$ was $57 \%, \mathrm{~L} 4-5\left(<42^{\circ}\right)$ was $47.8 \%$, and L5S1 $\left(<45^{\circ}\right)$ was $35.6 \%$. FT was noted to be $30.8 \%$ at L3 -4 , $35.6 \%$ at L4-5, and $45.2 \%$ at L5-S1 as shown in Tables 1 and 2. There was a positive relationship observed between SAF and LDH at L4-5 and L5-S1 with a p-value of 0.02 $(<0.05)$. FT showed a positive association with LDH at L4 -5 ( $p$-value $=0.047$ ) but not at L3-4 or L5-S1.

In the DS group, 16 had degenerative listhesis at L3-4, 180 at $\mathrm{L} 4-5$, and 54 at L5-S1. SAF at L3-4 was $65 \%, \mathrm{~L} 4-5$ was $64.6 \%$, and L5-S1 was $49 \%$. FT was noted to be $32.8 \%$ at $\mathrm{L} 3-4,26.4 \%$ at $\mathrm{L} 4-5$, and $39.2 \%$ at $\mathrm{L} 5-\mathrm{S} 1$ as shown in Tables 3 and 4. SAF showed a positive relationship with a $p$-value of $<0.001$ at L4-5 but not at L3-4 or L5-S1. FT showed a significant relation at L4-5 ( $p$-value $<0.001)$ but

Table 1. Relationship between LDH and SAF, FT

\begin{tabular}{|c|c|c|c|c|c|c|c|c|c|}
\hline \multirow{2}{*}{ Level } & \multirow{2}{*}{ LDH } & \multicolumn{2}{|c|}{ Side of LDH } & \multicolumn{3}{|c|}{ Relation to SAF } & \multicolumn{3}{|c|}{ Relation to FT } \\
\hline & & Right & Left & Yes & No & $p$-value & Yes & No & $p$-value \\
\hline L3-4 & 17 & 10 & 7 & 12 & 5 & 0.089 & 6 & 11 & 0.225 \\
\hline L4-5 & 135 & 62 & 73 & 81 & 54 & 0.020 & 56 & 79 & 0.047 \\
\hline L5-S1 & 98 & 47 & 51 & 38 & 60 & 0.026 & 53 & 45 & 0.419 \\
\hline
\end{tabular}

LDH, lumbar disc herniation; SAF, sagittally aligned facet; FT, facet tropism.

Table 2. Showing average facetal angles, SAF and facet tropism at three lower lumbar levels in group lumbar disc herniation

\begin{tabular}{lcccccc} 
& Facet angle (min) & Facet angle (max) & Mean facet angle & SAF & Tropism present & Tropism absent \\
L3-4 (right) & 11.9 & 63.65 & $35.27 \pm 9.89$ & $135^{\text {a) }}$ & 77 & 173 \\
L3-4 (left) & 11.55 & 63.64 & $34.77 \pm 9.94$ & $150^{\text {a) }}$ & $111^{\text {a) }}$ & 89 \\
\hline L4-5 (right) & 13.22 & 73.5 & $42.17 \pm 11.47$ & $128^{\text {a) }}$ & 161 \\
L4-5 (left) & 12.0 & 74.23 & $41.89 \pm 11.37$ & $88^{\text {a) }}$ & 113 \\
L5-S1 (right) & 17.0 & 91.04 & $49.32 \pm 12.01$ & $90^{\text {a) }}$ & 137 \\
L5-S1 (left) & 25 & 92.18 & $49.05 \pm 11.56$ & & & \\
\hline
\end{tabular}

Values are presented as number or mean \pm standard deviation.

SAF, sagittally aligned facet.

a) Denotes calculation of SAF based on angles of less than 36 at L3-4; 42 at $L 4-5$ and 45 at L5-S1.

Table 3. Relationship between DS and SAF, FT

\begin{tabular}{lrrrrrrrr} 
& & \multicolumn{3}{c}{ Relation to SAF } & & \multicolumn{3}{c}{ Relation to FT } \\
\cline { 3 - 8 } & DS & Yes & No & $p$-value & Yes & No & $p$-value \\
L3-4 & 16 & 10 & 6 & 0.785 & 6 & 10 & 0.173 \\
L4-5 & 180 & 132 & 48 & $<0.001$ & 54 & 126 & $<0.001$ \\
L5-S1 & 54 & 26 & 37 & 0.785 & 22 & 32 & 0.173 \\
\hline
\end{tabular}

DS, degenerative spondylolisthesis; SAF, sagittally aligned facet; FT, facet tropism. 
Table 4. Showing average facetal angles, SAF and facet tropism at three lower lumbar levels in group degenerative spondylolisthesis

\begin{tabular}{|c|c|c|c|c|c|c|}
\hline & Facet angle (min) & Facet angle (max) & Mean facet angle & SAF & Tropism present & Tropism absent \\
\hline L3-4 (right) & 9.98 & 67.59 & $33.23 \pm 11.74$ & $159^{\text {a) }}$ & 82 & 168 \\
\hline L3-4 (left) & 8.05 & 75.54 & $32.8 \pm 10.56$ & $166^{\text {a) }}$ & & \\
\hline L4-5 (right) & 11.12 & 79.08 & $27.86 \pm 12.16$ & $165^{\text {a) }}$ & 66 & 184 \\
\hline L4-5 (left) & 11.9 & 70.98 & $37.63 \pm 11.52$ & $158^{\text {a) }}$ & & \\
\hline L5-S1 (right) & 3.0 & 77.0 & $45.99 \pm 13.9$ & $124^{\text {a) }}$ & 98 & 152 \\
\hline L5-S1 (left) & 12.58 & 90.1 & $45.2 \pm 13.21$ & $121^{\text {a) }}$ & & \\
\hline
\end{tabular}

Values are presented as number or mean \pm standard deviation.

SAF, sagittally aligned facet.

a)Denotes calculation of SAF based on angles of less than 36 at L3-4; 42 at $L 4-5$ and 45 at L5-S1.

Table 5. Table showing conclusions of various studies with respect to the parameters studied and the modality used for study (CT/MRI)

\begin{tabular}{|c|c|c|c|}
\hline Author & $\mathrm{CT} / \mathrm{MRI}$ & Relation & Conclusion \\
\hline Farfan and Sullivan [6] & CT & $\mathrm{FA} / \mathrm{LDH}+$ & FT is associated with LDH \\
\hline Cyron and Hutton [7] & CT & $\mathrm{FA} / \mathrm{LDH}+$ & Facets with FT rotate towards the side of more oblique facet \\
\hline Van Schaik et al. [8] & CT & $\mathrm{FA} / \mathrm{LDH}+$ & Facets with FT rotate towards the side of more oblique facet \\
\hline Adams and Hutton [9] & Cadaver & FA/FT/LDH - & Torsion is unimportant in the etiology of lumbar disc degeneration and LDH \\
\hline Chadha et al. [12] & MRI & $\mathrm{FT} / \mathrm{LDH}+$ & FT is associated with LDH at L5 S1 not L4-L5 \\
\hline Ishihara et al. [10] & CT & $\mathrm{FT} / \mathrm{LDH}+$ & $\mathrm{FT}$ is radiological feature of $\mathrm{LDH}$ \\
\hline Karacan et al. [11] & CT & $\mathrm{FT} / \mathrm{LDH}+$ & LDH is associated with asymmetry and sagittalisation of facets \\
\hline Park et al. [13] & $\mathrm{MRI}$ & $\mathrm{FT} / \mathrm{LDH}+$ & Degree of FT distinguishes development of far lateral vs. posterolateral LDH \\
\hline Wang and Zhou [14] & $\mathrm{MRI}$ & FT/LDH - & FT is not associated with LDH \\
\hline Cassidy et al. [15] & CT & FT/LDH - & FT is not associated with LDH \\
\hline Vanharanta et al. [4] & CT & $\mathrm{FT} / \mathrm{LDH}-$ & FT is not associated with LDH \\
\hline Hagg and Wallner [16] & CT & FT/LDH - & FT is not associated with LDH \\
\hline Lee et al. [17] & CT & FT/LDH - & FT did not influence development of LDH \\
\hline Ko and Park [18] & CT & FT/LDH - & FT has no role in LDH \\
\hline Kunakornsawat et al. [19] & MRI & FT/LDH - & No relationship between FT and LDH \\
\hline Grobler et al. [20] & CT & $\mathrm{FA} / \mathrm{DS}+$ & DS is predisposed by developmental sagittal facets of $L 4-5$ \\
\hline Boden et al. [21] & CT & $\mathrm{FA} / \mathrm{DS}+$ & Sagittally oriented facets are associated with DS \\
\hline Cinotti et al. [22] & CT & FA/DS + & Sagittally oriented facets are associated with DS \\
\hline Kim and Lee [23] & CT & $\mathrm{FA} / \mathrm{DS}+$ & Patients with narrow facets more likely to develop DS \\
\hline Nagaosa et al. [24] & X-ray & FA/DS + & Horizontalization of facets is a risk factor for DS \\
\hline Sharma et al. [25] & Biomech & $\mathrm{FA} / \mathrm{DS}+$ & Sagittally oriented facets are associated with DS \\
\hline
\end{tabular}

CT, computed tomography; MRI, magnetic resonance imaging; FA, facet angle; LDH, lumbar disc herniation; FT, facet tropism; DS, degenerative spondylolisthesis.

did not show positive association at L3-4 and L5-S1.

\section{Discussion}

Facet joint orientation seems to play an important role in lumbar biomechanics. Asymmetry in the orientation of the facet joints is estimated to occur in $10 \%-70.5 \%$ of the population [5]. Our study revealed an overall incidence of FT of $52.5 \%$ (55.8\% in the DS group and $49.2 \%$ in the $\mathrm{LDH}$ group) in the 500 spinal units studied. The severity of FT increased from L3-L4 (32.8\% in the DS group and 30.8\% in the LDH group) to L5-S1 (39.8\% in the DS group and 
$45.2 \%$ in the LDH group). The intervertebral disc and both facet joints make a three-joint complex, each structure affecting the biomechanics of the other (coupling effect). Theoretically, facet joints share load mainly during compression and extension of the lumbar spine. They also protect the disc from excessive rotational strain and anterior shear forces. It therefore has been postulated that a more sagittal alignment of the joint leads to anterior gliding due to a reduced resistance to anterior shear forces [6-8]. These forces are particularly large in the lower two segments of the lumbar spine, as lordosis and horizontal vertebral tilt increase. The presence of coronal orientation at the L5S1 facet joint compared to L3-4 and L4-5 indicates the natural mechanism for overcoming these forces. Our study findings were consistent with regard to the presence of more SAFs at L3-4 (57\% in the DS group and 65\% in the LDH group) and coronally oriented facets at L5-S1 (35.6\% in the DS group and $49 \%$ in the LDH group).

\section{Relation between sagittally aligned facet and lumbar disc herniation}

Studies relating SAF and the development of LDH have yielded variable results (Table 5). Having more SAF joints offers little or no mechanical resistance to rotational forces, resulting in the inability to protect the disc from injury. Farfan and Sullivan [6] concluded that a more obliquely oriented facet would allow increased rotation toward the oblique side. In a cadaver-based study, Cyron and Hutton [7] noted that specimens with tropism were inclined to rotate toward the more oblique facet when an axial load was placed on the spine segment. A computed tomography (CT) scan study by van Schaik et al. [8] revealed that with greater degrees of asymmetry, there was an increased incidence of unilateral disc protrusion toward the side of the more coronally oriented joints. Although a majority of studies correlate the occurrence of disc herniation toward the more obliquely oriented facets, a few studies, such as that of Adams and Hutton [9] demonstrated that this angular rotation was not significant in the etiology of disc prolapse. However, in the present study, a significant correlation was observed between the side of SAF joint and LDH at L4-5 and L5-S1 levels. The L3-4 level did not demonstrate association with SAF or FT. We noted that there was a tendency for the occurrence of disc prolapse on the more sagittal side whenever there happened to be a combination of sagittal and coronal facets at the same level especially at L4-5 but not at L3-4 or L5-S1. We hypothesize that whenever the lower lumbar spine is loaded during flexion-extension, the more oblique side resists the angular motion, and the more SAF allows angular motion as well as rotation in order to compensate for the other facet. Thus, this rotational movement indirectly applies tensile stress on the annulus of the disc, leading to prolapse on the sagittal side (Fig. 1B).

\section{Relation between facet tropism and lumbar disc her- niation}

A similar controversy exists as regards the correlation between FT and LDH (Table 5). Farfan and Sullivan [6] were the first to suggest the correlation between FT and the development of LDH. Studies by Ishihara et al. [10] and Karacan et al. [11] further reported the same association. Recent studies by Chadha et al. [12] also suggested that FT is associated with LDH at the L5-S1 motion segment but not at the L4-L5 level. In this study, they found that the disc has herniated toward the sagittally oriented facet in 13 out of 19 cases with tropism; however, the sample size was small and insufficient. The degree of FT has been reported to play a role in the development of posterolateral as well as far-lateral disc herniation in a study by Park et al. [13]. However, several studies have disproved this hypothesis $[4,14-19]$. Our study demonstrated that FT is related to LDH at the L4-5 level but not at L3-4 or L5-S1.

\section{Relation between sagittally aligned facet and degen- erative spondylolisthesis}

The mobility between two adjacent motion segments is determined by the orientation of the plane of facet joint articulations. In comparison with other portions of the spine, facet joints in the lower lumbar spine have a closer orientation to the coronal plane and are more rigid during lateral bending [20]. Previous reports have demonstrated that facet orient ation is significantly associated with DS [21-29]. In an MRI-based study, Boden et al. [21] reported that both the left and the right facet joints were more sagittally oriented in patients with DS. Additionally, some of these studies reported that, in patients with DS, the transverse plane of facet joints was more sagittally oriented. Our study found that the SAF joint was significantly associated with DS only at L4-5, which was the most commonly affected level. In contrast to other studies, the L3-4 and L5- 
S1 levels did not demonstrate any positive association.

\section{Relation between facet tropism and degenerative spondylolisthesis}

The relationship between FT and DS remains a debatable issue. Berlemann et al. [30] reported that facet joint asymmetry does not seem to play a major role in the development of DS. A study by Kong et al. [31] found no association between FT and translational segmental motion. In an MRI-based study, Grannum et al. [32] suggested that sagittally oriented facets are not associated with the causation of DS. However, a recent study by Pichaisak et al. [29] reported a positive association between SAF and FT and the development of DS [33]. Our study demonstrated that the L4-5 level appears to be related to FT, but no significant relationship was observed between FT and DS at L3-4 and L5-S1.

\section{Limitations and strengths}

There were several limitations with regard to the current study. Initially, this was a cross-sectional observational study conducted only on surgically treated patients. The primary source of error could be in the identification of the reference plane and the margins of the facet joint, which is operator-dependent. The characteristic of hypertrophic 'lipping' of the superior facet can lead to errors in the measurement of facet joint angle; nevertheless, an established method was used for measurement, with proven consistency through interobserver reliability studies. The discrepancy in SAF or FT cutoff angles has been described to be varied in literature, and we have used only one methodology, which is the only applicable approach. Neither did we include sagittal balance, lumbar lordosis, other pelvic and angular parameters, body mass index, and other demographic features that may individually influence the development of LDH or DS. Although the measurement of facet joint angle from an MRI axial T2weighted image is less accurate than that from a CT image, we found the reliability of this measurement method was comparable and consistent with several previous studies. The strengths of the present investigation include the use of a large sample size. We have analyzed the facet orientation and tropism in each level and side of disc herniation, highlighting new concepts that can be useful for future longitudinal studies. Many previous studies have used the adjacent segment as a control to determine the results. However, we have used the same segment bearing a greater significance than the adjacent segment controls in performing comparisons. The measurement technique was highly reproducible, and the actual measurements were consistent with values reported in several previous studies whereby CT scans were used. Our methodology provided normative data as well as evidence of the clinical relevance for further studies on the orientation of the lumbar facet joint and FT.

\section{Conclusions}

In the present study, we found that the L4-5 level was significantly associated with SAF and FT in LDH and DS. SAF at L5-S1 demonstrated a positive association with LDH. These findings can provide useful information for future longitudinal studies. Nevertheless, further randomized epidemiological studies are needed to confirm such associations and also to elucidate the possible causes for such phenomenon.

\section{Conflict of Interest}

No potential conflict of interest relevant to this article was reported.

\section{References}

1. Putti V. New conceptions in the pathogenesis of sciatic pain. Lancet 1927;2:53-60.

2. Brailsford JF. Deformities of the lumbosacral region of the spine. Br J Surg 1929;16:562-627.

3. Noren R, Trafimow J, Andersson GB, Huckman MS. The role of facet joint tropism and facet angle in disc degeneration. Spine (Phila Pa 1976) 1991;16:530-2.

4. Vanharanta H, Floyd T, Ohnmeiss DD, Hochschuler $\mathrm{SH}$, Guyer RD. The relationship of facet tropism to degenerative disc disease. Spine (Phila Pa 1976) 1993;18:1000-5.

5. Murtagh FR, Paulsen RD, Rechtine GR. The role and incidence of facet tropism in lumbar spine degenerative disc disease. J Spinal Disord 1991;4:86-9.

6. Farfan HF, Sullivan JD. The relation of facet orientation to intervertebral disc failure. Can J Surg 1967;10:179-85.

7. Cyron BM, Hutton WC. Articular tropism and sta- 
bility of the lumbar spine. Spine (Phila Pa 1976) 1980;5:168-72.

8. Van Schaik JP, Verbiest H, Van Schaik FD. The orientation of laminae and facet joints in the lower lumbar spine. Spine (Phila Pa 1976) 1985;10:59-63.

9. Adams MA, Hutton WC. The relevance of torsion to the mechanical derangement of the lumbar spine. Spine (Phila Pa 1976) 1981;6:241-8.

10. Ishihara $H$, Matsui $H$, Osada R, Ohshima $H$, Tsuji $\mathrm{H}$. Facet joint asymmetry as a radiologic feature of lumbar intervertebral disc herniation in children and adolescents. Spine (Phila Pa 1976) 1997;22:2001-4.

11. Karacan I, Aydin T, Cidem M, Karamehmetoglu SS. Alteration of facet joint orientation and asymmetry with time in lumbar disc herniation. Neurosurg Q 2006;16:135-8.

12. Chadha M, Sharma G, Arora SS, Kochar V. Association of facet tropism with lumbar disc herniation. Eur Spine J 2013;22:1045-52.

13. Park JB, Chang H, Kim KW, Park SJ. Facet tropism: a comparison between far lateral and posterolateral lumbar disc herniations. Spine (Phila Pa 1976) 2001;26:677-9.

14. Wang H, Zhou Y. Facet tropism: possible role in the pathology of lumbar disc herniation in adolescents. J Neurosurg Pediatr 2016;18:111-5.

15. Cassidy JD, Loback D, Yong-Hing K, Tchang S. Lumbar facet joint asymmetry: intervertebral disc herniation. Spine (Phila Pa 1976) 1992;17:570-4.

16. Hagg O, Wallner A. Facet joint asymmetry and protrusion of the intervertebral disc. Spine (Phila Pa 1976) 1990;15:356-9.

17. Lee DY, Ahn Y, Lee SH. The influence of facet tropism on herniation of the lumbar disc in adolescents and adults. J Bone Joint Surg Br 2006;88:520-3.

18. Ko HY, Park BK. Facet tropism in lumbar motion segments and its significance in disc herniation. Arch Phys Med Rehabil 1997;78:1211-4.

19. Kunakornsawat S, Ngamlamaidt K, Tungsiripat $\mathrm{R}$, Prasartritha T. The relationship of facet tropism to lumbar disc herniation. J Med Assoc Thai 2007;90:1337-41.

20. Grobler LJ, Robertson PA, Novotny JE, Pope MH. Etiology of spondylolisthesis: assessment of the role played by lumbar facet joint morphology. Spine (Phila Pa 1976) 1993;18:80-91.

21. Boden SD, Riew KD, Yamaguchi K, Branch TP,
Schellinger D, Wiesel SW. Orientation of the lumbar facet joints: association with degenerative disc disease. J Bone Joint Surg Am 1996;78:403-11.

22. Cinotti G, Postacchini F, Fassari F, Urso S. Predisposing factors in degenerative spondylolisthesis: a radiographic and CT study. Int Orthop 1997;21:337-42.

23. Kim NH, Lee JW. The relationship between isthmic and degenerative spondylolisthesis and the configuration of the lamina and facet joints. Eur Spine J 1995;4:139-44.

24. Nagaosa Y, Kikuchi S, Hasue M, Sato S. Pathoanatomic mechanisms of degenerative spondylolisthesis: a radiographic study. Spine (Phila Pa 1976) 1998;23:1447-51.

25. Sharma M, Langrana NA, Rodriguez J. Role of ligaments and facets in lumbar spinal stability. Spine (Phila Pa 1976) 1995;20:887-900.

26. Sato K, Wakamatsu E, Yoshizumi A, Watanabe N, Irei O. The configuration of the laminas and facet joints in degenerative spondylolisthesis: a clinicoradiologic study. Spine (Phila Pa 1976) 1989;14:1265-71.

27. Dai L, Jia L. Role of facet asymmetry in lumbar spine disorders. Acta Orthop Belg 1996;62:90-3.

28. Fitzgerald JA, Newman PH. Degenerative spondylolisthesis. J Bone Joint Surg Br 1976;58:184-92.

29. Pichaisak W, Chotiyarnwong C, Chotiyarnwong P. Facet joint orientation and tropism in lumbar degenerative disc disease and spondylolisthesis. J Med Assoc Thai 2015;98:373-9.

30. Berlemann U, Jeszenszky DJ, Buhler DW, Harms J. Facet joint remodeling in degenerative spondylolisthesis: an investigation of joint orientation and tropism. Eur Spine J 1998;7:376-80.

31. Kong MH, He W, Tsai YD, et al. Relationship of facet tropism with degeneration and stability of functional spinal unit. Yonsei Med J 2009;50:624-9.

32. Grannum S, Torrie PA, Miller A, Harding IJ. Risk factors for the development of a mobile degenerative spondylolisthesis at L4-L5. Spine Deform 2015;3:98104.

33. Samartzis D, Cheung JP, Rajasekaran S, et al. Critical values of facet joint angulation and tropism in the development of lumbar degenerative spondylolisthesis: an international, large-scale multicenter study by the AOSpine Asia Pacific Research Collaboration Consortium. Global Spine J 2016;6:414-21. 